\title{
Intimacy as a Distinct Construct: Validating the Intimacy Scale among Older Adults of Residential Care Homes in Hong Kong
}

\author{
Wallace C.H. Chan", Siu M. To and Karen L.Y. Wong \\ Department of Social Work, The Chinese University of Hong Kong, Hong Kong, China
}

\begin{abstract}
Objectives: Intimacy is a construct that has received limited attention in the Chinese context. This study aimed to validate the Intimacy Scale among older adults in residential care homes in Hong Kong.

Method: Seventy-eight Chinese older adults were invited to respond to the Chinese version of the scale and other measurements validating the scale.

Results: The scale demonstrated good internal consistency and item-total correlation. Correlations supported the construct validity of the scale: significant positive correlations were found between perceived intimacy with the family caregiver and life satisfaction, presence of meaning and perceived family support. No correlation was found between intimacy and frequency of the family caregiver's visit.

Conclusion: We found the Intimacy Scale to be a valid measurement for assessing the quality of the relationship between older adults and family caregivers in the Hong Kong Chinese context. Assessing how older adults perceive the level of intimacy with family caregivers is important for helping professionals when working with older adults and their family members.
\end{abstract}

Keywords: Caregiver, Chinese, elderly, family, intimacy, relationship.

\section{INTRODUCTION}

In Hong Kong, as in many developed economies, life expectancy is increasing. Older adults may receive care and support from family members, especially when their health deteriorates. The caregiving and care-receiving processes may have a great impact on the relationship between older adults and their family caregivers [1-3]. Yet, the literature on gerontology seems to focus on the stress and burden of family caregivers $[4,5]$ and the frequency and effect of support given by family caregivers on older adults' psychological well-being [6]. Relatively little attempt has been made to explore older adults' perceived quality of relationship with their family caregivers. This is a notable omission, because the identification of a deep sentimental relational connection with family caregivers, such as spouse and adult children, can be of great importance for older adults in the caregiver-care receiver relationship. The literature focusing on family relations attempts to discuss the quality of the relationship between older adults and family members. For example, some studies suggest that a better relationship with the family is associated with better subjective well-being, such as the life satisfaction and happiness of older adults [7-11]. However, previous studies mainly examine the quality of the relationship from the perspective of the family caregivers. For example, Townsend and Franks [12] examined the quality of the relationship (e.g.

*Address correspondence to this author at the $4 / \mathrm{F}$, T.C. Cheng Building, United College, The Chinese University of Hong Kong, Shatin, Hong Kong. Tel: [852] 3943 1518; Fax: [852] 2603 5018;

E-mail: chchan@swk.cuhk.edu.hk emotional closeness and conflicts) from the perception of spousal caregivers and found that emotional closeness may mediate the impact of care receivers' cognitive impairment on the subjective caregiving effectiveness and conflicts in the spousal relationship. One exception is the study of Peters, Hooker, and Zvonkovic [13]. They addressed the views of older parents and highlighted their ambivalence in the relationship with adult children (e.g. they hope to have more quality time and help from adult children but understand that they have constraints in finance, work, and family).

One area that may reflect the quality of the family relationship is the level of intimacy. Assessing the intimacy between older adults and their family caregivers in the context of caregiving and care receiving is crucial, especially when caregiving may be perceived as stressful [14]. With a better understanding of the level of intimacy between older adults and their family caregivers, we may better develop appropriate intervention to enhance this intimacy, which is essential for improving the well-being of older adults. Therefore, the following parts of this article focus on the concept of intimacy, the associations between intimacy and other factors, and the relevance of validating a scale that measures intimacy among Chinese older adults living in residential homes in Hong Kong.

\section{CONCEPT OF INTIMACY}

The concept of intimacy is often discussed in loving relationships, which refers to the feelings of closeness, connectedness and bondedness [15]. Establishing intimacy is 
also considered by Erikson the developmental task of young adults [16]. Putting this concept in the context of older adults, intimacy often refers to the quality of relationship, emotional closeness [17], and affiliation between older parents and their adult children [18]. For example, Johnson and Bursk [7] (p. 92) defined "family relations" between older adults and adult children as showing "openness of communication between them, their enjoyment of each other's company, their ability to count on each other". Walker and Thompson [17] (p. 842-843) gave a comprehensive definition to the concept by summarizing the elements of intimacy suggested by the literature: "affection, altruism, enjoyment or satisfaction, a feeling that the relationship is important, openness or honesty, respect for the partner and acceptance of that person's ideas and criticism, solidarity, and a temporal commitment or sense of the certainty of the relationship".

\section{INTIMACY AS A DISTINCT CONSTRUCT}

Intimacy may have received less attention than is expected in studies of older adults. One reason is that the construct of intimacy may often be confused with similar constructs in gerontological studies, such as social support and frequency of contact, when understanding family caregiving of older adults. For example, relationships between older adults and family caregivers are often understood by the social support the older adults receive $[6$, 9]. The literature generally classifies social support into instrumental, referring to practical support (e.g. cleaning), and emotional $[6,9,19]$. However, in the context of family caregiving, intimacy is a distinct construct different from social support, which may reflect the quality of relationship between family caregivers and older adults. For example, Merz and Huxhold [9] showed that receiving instrumental support from kin may be associated with poorer subjective well-being of older adults, but a good-quality relationship between kin and the older adults may moderate this negative association.

Similarly, relationships between family caregivers and older adults are often understood by the quantity of contact $[6,17,20]$. For example, in the Hong Kong Chinese context of residential care homes, frequency of visits from family members is considered a major indicator of the quality of relationship with older adults. Walker and Thompson [17] pointed out that it was common in the literature for intimacy to be measured by frequency of contact, possibly because intimacy is an abstract concept to measure, whereas frequency of contact is easy to quantify. However, they showed that, for middle-aged daughters and older mothers, frequency of contact was not associated with intimacy in many situations, particularly from the perspective of the older mothers. Also, frequency of contact and another concept, exchange of aid, accounted for only a small proportion of variance of the concept of intimacy. Intimacy is therefore believed to be a distinct construct worthy of further exploration among older adults, especially in the context of family caregiving.

Intimacy of older adults may be associated with different factors, such as meaning in life and gender [11,21]. For example, Depaola and Ebersole [21] found that older adults living in nursing homes regarded family relationships as the most significant source of meaning in life. Intimacy with family members is thus expected to be positively correlated with meaning in life as perceived by older adults. Intimacy was also suggested to be related to gender. Ward [11] suggested that women are more likely than men to report maximum relationship quality. Brown and Amatea [22] indicated that women are more expressive about their emotions and more prepared to disclose their emotions in depth, influenced by the traditional gender division of labor and experiences while growing up. Therefore, older female adults are expected to report greater intimacy with family members than are their male counterparts.

\section{VALIDATING THE CONSTRUCT OF INTIMACY AMONG CHINESE OLDER ADULTS LIVING IN RESIDENTIAL CARE HOMES IN HONG KONG}

According to Lee [23-25], in Chinese culture, older adults' admission to a residential care home for care and living is often linked to negative feelings, such as being abandoned or rejected like a burden by family members. Lee said that older adults regarded their admission as a loss of ties with family members and perceived this as their failure as parents, failure of their adult children, and loss of respect from family. The traditional Chinese belief that children should care for and support the older adults at home may greatly influence these perceptions. This may also be a source of fear, anxiety, and stress for older adults in residential homes. In such a cultural context, assessing intimacy between Chinese older adults who live in residential homes and their family members would be of great significance to the well-being of the older adults.

In the West, one way to measure the relationship quality between older adults, who are often care receivers, and adult children, who are often caregivers, is to use the Intimacy Scale, developed by Walker and Thompson [17]. This scale measures intimacy directly, without confusing the concept with related concepts such as social support and frequency of visit. It also covers a wide range of elements of intimacy. Other authors exploring relationships not only between mother and daughter [26-28] but also between father and daughter or between friends [29-35] have widely adopted this scale. However, researchers outside the Western world have rarely used it, such as in the Chinese context, possibly because no suitable Chinese version of the scale was available. Therefore, this study aimed to validate the Intimacy Scale developed by Walker and Thompson [15] among Chinese older adults who live in residential care homes in Hong Kong. To our knowledge, our study is the first validating this scale in the Chinese context, which may help to examine its cross-cultural applicability in measuring quality of relationship between older adults and their family caregivers [36].

\section{MATERIALS AND METHODOLOGY}

Our study was a part of a larger study exploring the relationship between older adults living in residential care homes and their family caregivers in Hong Kong. The larger study consisted of a one-year, three-time-point longitudinal survey with the older adults, focus groups with the family caregivers, and a cross-sectional survey with the family 
caregivers. The data in this paper were elicited from the last phase of the longitudinal survey with the older adults. Tieu, Konnert, and Wang [37] suggested that, in research with Chinese older adults, it was common for participants to give answers they perceived to be socially desirable in order to "save face". With the assumptions that the problem of social desirability should be smaller when there is stronger rapport between the interviewer and the older adult, and rapport should be stronger by increasing the number of contacts via interviews, the answers of the older adults in the last phase of the study should be least influenced by social desirability. Therefore, the data of the last phase were used for analysis.

\section{Participants and Procedures}

Our targeted samples are older adults who receive care in residential care homes. We recruited them via four residential care homes in Hong Kong. A non-governmental organization runs these homes, and the government subsidizes them. Residents are older adults who were assessed to have moderate to severe impairment by the Minimum Data Set for Home Care [MDS-HC]. Because the questionnaire may be cognitively difficult for some of the older adults to complete, social workers in the residential care homes helped select potential participants who cognitively functioned well. For potential participants with MMSE results (Mini-Mental State Examination) [38], those scoring 20 or above, or 19 or below but were able to communicate meaningfully, were selected. The social workers helped select those without MMSE results who were cognitively fit, according to their daily observation. They identified 250 potential participants as the sampling frame. Inviting social workers to screen suitable participants is common in studies on older adults living in residential care homes [39].

Of the 250 potential participants, the social workers helped exclude those who were unfit (e.g. had dementia, were physically fragile or hearing impaired] or had a language barrier. The social workers approached the remaining potential participants and introduced them to the study. Because there was a relatively small number of potential cognitively and physically suitable participants, and in consideration of time and costs, we used convenience sampling, which is common in studies on older adults [37].

We interviewed 78 residents. The sample was relatively small, particularly compared to the one suggested by Walker and Thompson [17]. One reason is that fewer older adults in residential homes were physically or cognitively suitable for interviewing than expected.

Research assistants interviewed participants between October and November 2012 (the last phase of a three-time point longitudinal survey). Participants responded to the items of scales by choosing the options that best represented them. Each interview lasted about an hour. We sought written or verbal consent before the participants started completing the questionnaire. We also sought ethics approval for this study from the Research Ethics Committee of the first author's affiliating university.

\section{Measures}

The measures we examine in this paper include the Intimacy Scale, Satisfaction with Life Scale (SWLS), Meaning in Life Questionnaire (MLQ), Family subscale of Multidimensional Scale of Perceived Social Support, and Frequency of Visit (single item).

The Chinese Version of the Intimacy Scale. (CIS) Walker and Thompson [17] developed the Intimacy Scale to measure the intimacy between mother and daughter. Intimacy is the affection and emotional closeness in a relationship. This scale was also used to measure intimacy between older mothers (care receivers) and adult daughters (caregivers), which indicates the quality of their relationship [40]. It consists of 17 items, each rated on a scale of 1 to 7 . A higher score represents greater intimacy/affection. Walker and Thompson [17] reported good reliability of this scale in the original study (from 0.91 to 0.97 ). The significant positive relationship between intimacy and frequency of contacts between mothers and daughters [17] showed construct validity.

Based on the experience in the pilot study (we interviewed another 30 older adults), we found that older adults had more trouble responding to items of this scale when we used the original rating (range: 1-7). We therefore modified the rating of this scale to 1 to 5 . We summed up the items to generate a total score. The total potential range of the scale of our study was between 17 and 85. A higher score represents greater intimacy between the older adult and the family caregiver.

A research assistant translated the English version of this scale into Chinese and then the first and second authors back-translated it. Both the research assistant and the two authors are fluent in English and Chinese. We resolved discrepancies between the first translation and the back translation after discussion among the research assistant and two authors. We revised the wording and added examples for some items after the pilot test on 30 older adults living in a residential care home.

Item 1, "we want to spend time together", in Chinese culture is often interpreted to mean "we want to live together". We addressed this problem by adding an example: "she/he comes to the residential home and visits me, or we have a walk in the residential home". Some of the older adults in the pilot test had difficulty understanding the word "honest" in item 3, "we're honest with each other". We attempted to solve this problem by adding an example: "we do not lie to each other". And because some of the older adults in the pilot test also had trouble comprehending the word "unselfishness" in item 11, "there's a great amount of unselfishness in our relationship", we added an example: "we are prepared to make sacrifices for each other".

Satisfaction with Life Scale (SWLS). We used the SWLS to assess the general life satisfaction of older adults. Diener, Emmons, Larsen, and Griffin [41] developed the scale to measure the general life satisfaction of the participants. We chose it on the belief that life satisfaction 
should be based on individual judgment rather than on external criteria. SWLS measures one's life satisfaction according to personal judgment. An additional merit is that it was previously validated in an older adult sample, suggested in the paper on the development of SWLS. It includes 5 items, with a rating from 1 to 7 . Again, considering the potential difficulty for some participants to choose the best answer among the 7-point scale, we modified the ratings of SWLS to a 5-point scale, from 1 to 5 in this study and summed up the items to form a total score. The potential range of the scale of our study was between 5 and 25 . Higher scores represent higher life satisfaction. The SWLS in our study revealed good internal consistency with a Cronbach's alpha of 0.72 . The Chinese version in our study has been widely used in Hong Kong studies, for example, the one by Chan, Ungvari, Shek, and Leung [42].

Meaning in Life Questionnaire [MLQ]. Steger, Frazier, Oishi, and Kaler [43] developed The Meaning in Life Questionnaire. It consists of two subscales, Presence of Meaning and Search for Meaning. Each consists of 5 items, rated on a 7-point scale, from 1 to 7 . We summed up the items of each subscale to form a total score. A higher score represents a higher level of meaning (Presence subscale) or a greater desire to search for meaning (Search subscale). Each subscale of our study showed good internal consistency with Cronbach's alpha of 0.72 and 0.92 for the Presence subscale and Search subscale, respectively. Chan [44] validated the Chinese version of the MLQ (C-MLQ) in a Hong Kong Chinese student sample. Another study by Chan [45] explored its factor structure among Chinese caregivers. Our study used this validated version of the MLQ.

Family Subscale of Multidimensional Scale of Perceived Social Support. Zimet, Dahlem, Zimet, and Farley [46] developed the Multidimensional Scale of Perceived Social Support to measure a person's perceived social support from different sources. Intense discussion has taken place on the differentiation of different kinds of social support, but Zimet et al. [46] argued that social support from other people is a subjective concept, and the focus should be how one perceives the support. The full 12-item scale consists of three subscales, each measuring different perspectives of social support from family, friends, and significant others. We selected the Family subscale to assess family support from the family caregiver as the older adults in our study perceived it. Consistent with the original paper, the subscale in our study includes 4 items, with a rating from 1 to 7 . We summed up the items to form a total score. The potential range of the subscale of our study was between 7 and 28. A higher score represents a higher level of family support from the family caregiver as the older adult perceived it. The word "support" is defined by the interviewee. For example, it could be instrumental, emotional, and other types. The only exception is the item "I get the emotional help and support I need from my family", which restricts interviewees to respond to the emotional support they perceive as receiving from their family. The Family subscale of our study revealed good internal consistency with a Cronbach's alpha of 0.74. Chou [47] validated this subscale in a Hong Kong Chinese adolescent population, and we used the validated version for our study.
Frequency of Visit. We used a single item to measure how often the family caregiver visited the older adult. Available answer options include once a year, once or twice every six months, once or twice a month, once or twice a week, depends, and inapplicable.

\section{Data Analysis}

We conducted descriptive analyses to explore the demographics. We examined the internal consistency of the scale referring to Cronbach's alpha. We also examined itemtotal correlation. We examined construct validity of the scale by exploring the following relationships among constructs: i) relationship between older adults' perceived intimacy with the family caregiver and the life satisfaction of older adults, ii) relationship between intimacy and meaning in life of older adults, iii) relationship between intimacy and older adults' perceived family support from the family caregiver, iv) relationship between intimacy and frequency of the family caregiver's visit.

\section{RESULTS}

Demographics. According to Table 1, the majority of the 78 participants were female (67.95\%), and the mean age was $84.25(S D=7.99)$. Most were born in mainland China $(65.38 \%)$. The mean number of years living in Hong Kong is $66.25(S D=18.70)$. Of the 78 participants, $43.59 \%$ never received formal education; $26.92 \%, 25.64 \%$, and $20.51 \%$ were Protestant, had no religion, or worshipped ancestors respectively; and $61.54 \%$ were full-time workers before retirement. The majority $(64.10 \%)$ were widowed. The mean of number of children was $3.18(S D=2.43)$, and the mean number of grandchildren $4.44(S D=4.13)$. The mean of length of residency was 5.01 years $(S D=3.77)$. The majority $(70.51 \%)$ had their children as their family caregiver.

Descriptive Data of Different Measures. Table 2 presents the mean and standard deviation of the Intimacy Scale and each of the 17 items. The mean of the scale was $71.53(S D=13.50)$. According to the table, items 3 and 4 revealed relatively low means compared with other items, $3.77(S D=1.33)$ and $3.57(S D=1.15)$ respectively.

\section{Reliability}

Internal consistency. The Intimacy Scale showed good internal consistency. Cronbach's alpha is 0.97.

Item-total correlation. Referring to Table $\mathbf{2}$, all items demonstrate significant item-total correlations $(M=0.86)$, revealing moderate to high correlations and indicating that all items appropriately capture the construct of intimacy. The correlations of items 3 and 4 , both 0.69 , are relatively low compared with other items. Further discussion is in the Discussion section.

\section{Validity}

Construct validity. Table 3 reveals the correlations between the CIS and other scales validating the Intimacy Scale. All correlations support the construct validity of the 
Table 1. Demographics of older adults living in residential care homes $(\mathrm{N}=78)$.

\begin{tabular}{|c|c|c|}
\hline Variable & $N(\%)$ & $M(S D)$, Range \\
\hline \multicolumn{3}{|l|}{ Gender } \\
\hline Male & $25(32.05)$ & \\
\hline Female & $53(67.95)$ & \\
\hline Age & & 84.25 (7.99), 66-103 \\
\hline \multicolumn{3}{|l|}{ Place of Birth } \\
\hline Hong Kong & $20(25.64)$ & \\
\hline Mainland China & $51(65.38)$ & \\
\hline Other & $7(8.97)$ & \\
\hline Years living in Hong Kong & & $66.25(18.70), 7-93$ \\
\hline \multicolumn{3}{|l|}{ Education } \\
\hline No formal education & $34(43.59)$ & \\
\hline Primary school or below & $21(26.92)$ & \\
\hline Junior high school & $7(8.97)$ & \\
\hline High school & $5(6.41)$ & \\
\hline Tertiary education or higher & $5(6.41)$ & \\
\hline Other & $6(7.69)$ & \\
\hline \multicolumn{3}{|l|}{ Religion } \\
\hline Buddhism & $3(3.85)$ & \\
\hline Daoism & $13(16.67)$ & \\
\hline Catholic & $5(6.41)$ & \\
\hline Protestant & $21(26.92)$ & \\
\hline No religion & $20(25.64)$ & \\
\hline Ancestor worship & $16(20.51)$ & \\
\hline \multicolumn{3}{|l|}{ Last Occupation Before Retirement } \\
\hline Homemaker & $13(16.67)$ & \\
\hline Self-employed & $12(15.38)$ & \\
\hline Employer & $1(1.28)$ & \\
\hline Full-time worker & $48(61.54)$ & \\
\hline Part-time worker & $2(2.56)$ & \\
\hline Other & $2(2.56)$ & \\
\hline \multicolumn{3}{|l|}{ Marital Status } \\
\hline Single & $7(8.97)$ & \\
\hline Married & $20(25.64)$ & \\
\hline Divorced & $1(1.28)$ & \\
\hline Widowed & $50(64.10)$ & \\
\hline Number of children & & $3.18(2.43), 0-10$ \\
\hline Number of grandchildren & & 4.44 (4.13), $0-20$ \\
\hline Years of residence in the care home & & $5.01(3.77), 1-16$ \\
\hline \multicolumn{3}{|c|}{ Main Family Caregiver of Older Adult } \\
\hline Child & $55(70.51)$ & \\
\hline Grandchild & $4(5.13)$ & \\
\hline Spouse & $3(3.85)$ & \\
\hline Sibling & $2(2.56)$ & \\
\hline Other family member & $13(16.67)$ & \\
\hline Other & $1(1.28)$ & \\
\hline
\end{tabular}

scale. There is a positive and significant correlation between older adults' perceived intimacy with the family caregiver and the life satisfaction of the older adults $(r=0.40, p<0.01)$. We also found a positive and significant correlation between intimacy and the Presence subscale of C-MLQ of older adults $(r=0.25, p<0.05)$, but we found no significant correlation between intimacy and the Search subscale of CMLQ. There is also a positive and significant correlation between intimacy and older adults' perceived family support $(r=0.59, p<0.01)$. There is no significant correlation between intimacy and frequency of family caregiver's visit.

\section{DISCUSSION}

We found the Chinese version of the Intimacy Scale (CIS) to be reliable and valid in the current sample of Hong Kong Chinese older adults who lived in residential care homes. The internal consistency of CIS is very good, and the item-total correlations reflect that each item seems to correspond well to the overall construct of intimacy.

In the context of family caregiving, intimacy is a construct which refers to the emotional closeness between older adults (care receivers) and family members (caregivers) and can be used to indicate their quality of relationship. Findings of our study supported the construct validity of CIS by showing that the majority of correlations between CIS and other scales are in the expected directions. Ryff [48] identified that both middle-aged and older adults perceived the relationship with family members as the most important element in their psychological well-being. A study in Hong Kong also showed that older adults experienced higher level of life satisfaction when they received social support, including emotional support and appraisal support, from grandchildren [49]. Earlier studies also revealed that family relationships are often the major source of meaning in life among older adults [21, 50]. Our findings echo the findings in previous studies and confirmed the construct validity of CIS by showing that Intimacy was positively associated with well-being of older adults, as measured by life satisfaction and meaning in life in this study.

Similarly, the literature also examined the impact of quality of the pre-illness relationship with family caregivers, such as spouse, on caregiving and care receiving (e.g. caregiving burden, the quantity and quality of support) and suggested that better quality of relationship may ensure better support provided to older adults (care receivers) [51, 52]. Again, our findings echo the literature and thus support the construct validity of CIS, showing that intimacy, as an indicator of relationship quality between caregivers and care receivers, is positively associated with perceived family support in this study.

Unlike the Presence subscale of C-MLQ, the Search subscale of C-MLQ did not show any significant correlation with the CIS. A similar result was found in a study of long-term survivors, in which Dirksen [53] suggested that no correlation was found between search for meaning and intimacy. It is also consistent with the notion of "will to meaning" of logotherapy [54]. Will to meaning means that human beings have a basic striving for meaning in all circumstances. Therefore, regardless of how older adults perceived their intimacy with the family, they will continue to search for meaning. 
Table 2. Mean scores and standard deviation of the Intimacy Scale ${ }^{a}$ and its items, ${ }^{b}$ and item-total correlation of the Intimacy Scale.

\begin{tabular}{|c|c|c|}
\hline & $M(S D)$ & Item-Total Correlation \\
\hline She/he shows that she/he loves me. & $4.25(0.89)$ & $0.85^{* *}$ \\
\hline We're honest with each other, i.e., we do not lie to each other. & $3.77(1.33)$ & $0.69^{* *}$ \\
\hline We like each other. & $4.31(0.82)$ & $0.85^{* *}$ \\
\hline We respect each other. & $4.29(0.82)$ & $0.89^{* *}$ \\
\hline Our lives are better because of each other. & $4.31(0.89)$ & $0.90^{* *}$ \\
\hline We enjoy the relationship. & $4.30(0.89)$ & $0.93^{* *}$ \\
\hline She/he always thinks of my best interest. & $4.12(1.00)$ & $0.89^{* *}$ \\
\hline I'm lucky to have her/him in my life. & $4.43(0.80)$ & $0.81^{* *}$ \\
\hline She/he always makes me feel better. & $4.22(0.94)$ & $0.92^{* *}$ \\
\hline She/he is important to me. & $4.45(0.82)$ & $0.82^{* *}$ \\
\hline We love each other. & $4.32(0.83)$ & $0.93^{* *}$ \\
\hline I'm sure of this relationship. & $4.35(0.85)$ & $0.90^{* *}$ \\
\hline
\end{tabular}

Note: ${ }^{a}$ Potential range of score of Intimacy Scale is $17-85$; ${ }^{b}$ Potential range of score of each item of Intimacy Scale is 1-5; Language used in interviews of our study is Chinese. $* * p<0.01$

Our finding also show that older adults' perceived intimacy with family caregivers was not associated significantly with the frequency of visits of the family caregivers. This finding may support our assertion that intimacy is a distinct construct which measures the quality of relationship and is different from other indicators, such as frequency of visits, which only suggests quantity. A study in Hong Kong also supported this view by showing that older adults emphasized the quality of support their children give more than the quanity of support [e.g. frequency of visits and interactions] when looking at the issue of filial piety [55].

Table 3. Correlations between the Intimacy Scale and other scales.

\begin{tabular}{|c|c|}
\hline & Intimacy \\
\hline \hline Life Satisfaction & $0.40^{* *}$ \\
\hline Meaning (presence) & $0.25^{*}$ \\
\hline Meaning (search) & 0.17 \\
\hline Family Support & $0.59^{* *}$ \\
\hline Frequency of Visit & 0.08 \\
\hline${ }^{*} p<0.05 ; *^{*} p<0.01$. & \\
\hline
\end{tabular}

In view of the cultural context, we would like to highlight the relatively low mean scores and item-total correlations in item 3 ("we're honest with each other; i.e. we do not lie to each other") and item 4 ("we can accept each other's criticism of our faults and mistakes"]. A possible explanation is that, in Chinese culture, particularly in a sample of older adults, preservation of face is important [37]. Chinese older adults care not only about preservation of their own face but also of family members' face. Therefore, the Chinese older adults may tell "white lies" with the intention of saving the family caregiver's face. Also, it might be difficult for them to accept being criticized by family caregivers. This explains why the mean and item-total correlations are low for items 3 and 4 when compared with other items. This suggests that items 3 and 4 may not totally suit the understanding of intimacy in a Chinese older adult context. Future research may further investigate if these two items are relevant in measuring intimacy in the Chinese context.

Intimacy in the context of caregiving is a concept worthy of more attention in the Chinese context. As our findings show, the CIS may help to reveal the emotional connectedness and quality of relationship between family caregivers and older adults. In fact, intimacy may be something Chinese people often neglect in caregiving, as traditionally we emphasize obligation and responsibility in caregiving [56], especially when filial piety may imply adult children are expected to take care of their older parents in the Chinese culture [57]. Yet, studies have showed that Chinese family caregivers do not take care of their older adults only due to obligation and responsibility. They also emphasize the importance of affection with older adults as the motivation of their caregiving $[56,58]$. 
Therefore, the CIS could be an effective tool objectively and quantitatively measuring the intimacy between the older adult and the family caregiver, which may be culturally ignored in the caregiving of Chinese people. A residential care home is a multidisciplinary work setting, and the concept of intimacy is abstract and subjective. Therefore, the Intimacy Scale could be a scientific and objective measurement of intimacy and thus facilitate multidisciplinary collaboration. Professionals, including social workers, may intervene by improving the relationship between the older adult and the family caregiver. Social workers may work with them according to the level of intimacy perceived by the older adult, for example, facilitating expression of intimacy between the older adult and the family caregiver, improving support from the family caregiver to the older adult, or enhancing the older adult's appreciation of the family caregiver's caregiving contribution. The CIS should also facilitate comparison among different groups of older adults, not only those living in residential care homes but also those living in the community. Moreover, whereas there are programs aiming to enhance the relationship between the older adult and the family caregiver, the CIS could shed light on accurately measuring the effectiveness of these programs by checking whether or not the intimacy level perceived by the older adult has improved over time.

The study does have limitations. As suggested, participants might tend to choose answers that are socially desirable to them, because of the influence of Chinese culture. Although this paper attempts to minimize the problem by using the third phase of the data, future studies testing the Intimacy Scale in a Chinese older adult sample may minimize the problem further by including a social desirability scale [59]. Moreover, the study had a relatively small sample size $(\mathrm{N}=78)$, which is understandable due to the nature of our sample consisting of older adults living in residential care homes. Future studies of larger sample size can be conducted to further verify the psychometric properties of the CIS (e.g. the factor structure).

Furthermore, this study only examined the intimacy between the older adult and the family caregiver. Future studies may apply the validated CIS to examine the intimacy between the older adult and other people such as friends or their caregiverss such as staff of the residential care homes. There are studies that suggest that the relationship between older adults and these people may have significant effects on the well-being of older adults $[6,39]$.

\section{CONCLUSION}

In this study, the Chinese version of the Intimacy Scale was validated among Chinese older adults who lived in residential homes for elderly people. Intimacy is a distinct construct which reflects the quality of relationship between family members. By validating this scale in the Chinese context, it is hoped that future studies can further examine the importance of intimacy among family members, such as the intergenerational relationship between older adults and their adult children.

\section{CONFLICT OF INTEREST}

The authors confirm that this article content has no conflict of interest.

\section{ACKNOWLEDGEMENTS}

Declared none.

\section{REFERENCES}

[1] Walker AJ, Allen KR. Relationships between caregiving daughters and their elderly mothers. Gerontologist 1991; 31(3): 389-96.

[2] Davis LL, Gilliss CL, Deshefy-Longhi T, Chestnutt DH, Molloy M. The nature and scope of stressful spousal caregiving relationships. J Fam Nurs 2011;17(2): 224-40.

[3] Pinquart M, Sörensen S. Spouses, adult children, and children-inlaw as caregivers of older adults: A meta-analytic comparison. Psychol Aging 2011; 26(1): 1-14.

[4] Bevans M, Sternberg EM. Caregiving burden, stress, and health effects among family caregivers of adult cancer patients. JAMA [Online] 2012 Jan 25 [cited 2014]; 307[4]. Available from: http://jama.jamanetwork.com/article.aspx?doi=10.1001/jama.2012. 29

[5] Chan CLF, Chui EWT. Association between cultural factors and the caregiving burden for Chinese spousal caregivers of frail elderly in Hong Kong. Aging Ment Health 2011;15(4): 500-9.

[6] Cheng S-T, Lee CKL, Chow PK-Y. Social support and psychological well-being of nursing home residents in Hong Kong. Int Psychogeriatr 2010; 22(7): 1185-90.

[7] Johnson ES, Bursk BJ. Relationships between the elderly and their adult children. The Gerontologist 1977; 17(1): 90-6.

[8] Mancini JA, Blieszner R. Aging parents and adult children: Research themes in intergenerational relations. J Marriage Fam 1989; 51(2): 275-90.

[9] Merz E-M, Huxhold O. Wellbeing depends on social relationship characteristics: Comparing different types and providers of support to older adults. Ageing Soc 2010; 30(5): 843-57.

[10] Umberson D. Relationships with children: Explaining parents' psychological well-being. J Marriage Fam 1989; 51(4): 999-1012.

[11] Ward RA. Multiple parent-adult child relations and well-being in middle and later life. J Gerontol Soc Sci 2008; 63B(4): S239-S247.

[12] Townsend AL, Franks MM. Quality of the relationship between elderly spouses: Influence on spouse caregivers' subjective effectiveness. Fam Relat 1997; 46: 33-9.

[13] Peters CL, Hooker K, Zvonkovic AM. Older parents' perceptions of ambivalence in relationships with their children. Fam Relat 2006; 55(5): 539-51.

[14] Aronson J. Women's perspectives on informal care of the elderly: Public ideology and personal experience of giving and receiving care. Ageing Soc 1990; 10(1): 61-84.

[15] Sternberg RJ. Construct validation of a triangular love scale. Eur J Soc Psych 1997; 27(3): 313-35.

[16] Erikson EH. Identity and the life cycle: Selected papers. Psychol Issues 1959; 1: 1-171.

[17] Walker AJ, Thompson L. Intimacy and intergenerational aid and contact among mothers and daughters. J Marriage Fam 1983; 45(4): 841-9.

[18] Ohta M, Kai I. The cross-validity of the filial obligation scale. Arch Gerontol Geriatr 2007; 45(1):1-8.

[19] Yeung GTY, Fung HH. Social support and life satisfaction among Hong Kong Chinese older adults: Family first? Eur J Age 2007; 4(4): 219-27.

[20] Lee JJ, Lo SC. A survey of the residential care needs of older people. Hong Kong: Tung Wah Group of Hospitals 2005.

[21] Depaola SJ, Ebersole P. Meaning in life categories of elderly nursing home residents. Int J Aging Hum Dev. 1995; 40(3): $227-$ 36.

[22] Brown NM, Amatea ES. Love and intimate relationships: Journey of the heart. Philadelphia. PA: Brunner/Mazel 2000. 
[23] Lee DTF. Perceptions of Hong Kong Chinese elders on adjustment to residential care. J Interprof Care 2001; 15(3): 235-44.

[24] Lee DTF. Residential care placement: perceptions among elderly Chinese people in Hong Kong. J Adv Nurs 1997; 26(3): 602-7.

[25] Lee DTF. Transition to residential care: Experiences of elderly Chinese people in Hong Kong. J Adv Nurs 1999; 30(5): 1118-26.

[26] Koerner SS, Wallace S, Lehman SJ, Raymond M. Mother-todaughter disclosure after divorce: Are there costs and benefits? J Child Fam Stud 2002; 11(4): 469-83.

[27] Pratt CC, Jones-Aust L, Pennington D. Decision-making influence strategies of caregiving daughters and their elderly mothers. Fam Relat 1993; 42(4): 376-82.

[28] Smith LM, Hill EW, Mullis RL. Relational perceptions in motherdaughter attachments. Marriage Fam Rev 1998; 27(1-2): 37-49.

[29] Bulger MW, Wandersman A, Goldman CR. Burdens and gratifications of caregiving: Appraisal of parental care of adults with schizophrenia. Am J Orthopsychiatry 1993; 63(2): 255-65.

[30] Buote VM, Wood E, Pratt M. Exploring similarities and differences between online and offline friendships: The role of attachment style. Comput Hum Behav 2009; 25(2): 560-7.

[31] Campbell L, Butzer B, Wong J. The importance of the organization of partner knowledge in understanding perceptions of relationship quality and conflict resolution behavior in married couples. Pers Soc Psychol Bull 2008; 34(6): 723-40.

[32] Clark KJ, Kanoy K. Parents' marital status, father-daughter intimacy and young adult females' dating relationships. J Divorce Remarriage 1998; 29(1-2): 167-79.

[33] Cohen SP. Can pets function as family members? West J Nurs Res 2002; 24(6): 621-38.

[34] Cooney TM, Hutchinson MK, Leather DM. Surviving the breakup? Predictors of parent-adult child relations after parental divorce. Fam Relat 1995; 44(2): 153-61.

[35] Jewell TC, Stein CH. Parental influence on sibling caregiving for people with severe mental illness. Community Ment Health J 2002; 38(1): 17-33.

[36] Hook MK, Gerstein LH, Detterich L, Gridley B. How close are we? Measuring intimacy and examining gender differences. J Couns Develop 2003; 81(4): 462-72.

[37] Tieu Y, Konnert C, Wang J. Depression literacy among older Chinese immigrants in Canada: A comparison with a populationbased survey. Int Psychogeriatr 2010; 22(8):1318-26.

[38] Chiu HFK, Lee HC, Chung WS, Kwong PK. Reliability and validity of the Cantonese version of Mini-mental State Examination: A preliminary study. J Hong Kong Coll Psychiatr 1994; 4: 25-8.

[39] Bitzan JE, Kruzich JM. Interpersonal relationships of nursing home residents. Gerontologist 1990; 30(3): 385-90.

[40] Walker AJ, Pratt CC, Shin H-Y, Jones LL. Motives for parental caregiving and relationship quality. Fam Relat 1990; 39(1): 51-6.

[41] Diener E, Emmons RA, Larsen RJ, Griffin S. The satisfaction with life scale. J Pers Assess 1985; 49(1): 71-5.
[42] Chan GWL, Ungvari GS, Shek DTL, Leung JJP. Hospital and community-based care for patients with chronic schizophrenia in Hong Kong. Soc Psychiatry Psychiatr Epidemiol 2003; 38(4): 196203.

[43] Steger MF, Frazier P, Oishi S, Kaler M. The meaning in life questionnaire: Assessing the presence of and search for meaning in life. J Couns Psychol 2006; 53(1): 80-93.

[44] Chan WCH. Assessing meaning as outcome and coping: Validation of two meaning-related scales in Hong Kong. Unpublished manuscript 2012.

[45] Chan WCH. Factor structure of the Chinese version of the Meaning in Life Questionnaire in Hong Kong Chinese caregivers. Health Soc Work 2014; 39(3): 135-43.

[46] Zimet GD, Dahlem NW, Zimet SG, Farley GK. The multidimensional scale of perceived social support. J Pers Assess 1988; 52(1): $30-41$.

[47] Chou K-L. Assessing Chinese adolescents' social support: The multidimensional scale of perceived social support. Pers Ind Differ 2000; 28(2): 299-307.

[48] Ryff CD. In the eye of the beholder: Views of psychological wellbeing among middle-aged and older adults. Psychol Aging 1989; 4(2):195-210.

[49] Lou VWQ. Life satisfaction of older adults in Hong Kong: The role of social support from grandchildren. Soc Indic Res 2010; 95(3): $377-91$.

[50] Ebersole P, Depaola S. Meaning in life categories of later life couples. J Psychol 1987; 121(2): 185-91.

[51] Steadman PL, Tremont G, Davis JD. Premorbid relationship satisfaction and caregiver burden in dementia caregivers. J Geriatr Psychiatry Neurol 2007; 20(2): 115-9.

[52] Williamson GM, Shaffer DR. The Family Relationships in Late Life Project. Relationship quality and potentially harmful behaviors by spousal caregivers: How we were then, how we are now. Psychol Aging 2001; 16(2): 217-26.

[53] Dirksen SR. Search for meaning in long-term cancer survivors. J Adv Nurs 1995; 21(4): 628-33.

[54] Frankl VE. Man's search for meaning. Boston, MA: Beacon Press 2006.

[55] Cheng S-T, Chan ACM. Filial piety and psychological well-being in well older Chinese. J Gerontol B Psychol Sci Soc Sci 2006; 61(5): P262-P269.

[56] Holroyd E. Hong Kong Chinese daughters' intergenerational caregiving obligations: A cultural model approach. Soc Sci Med 2001; 53: 1125-34

[57] Lai DWL. Filial piety, caregiving appraisal, and caregiving burden. Res Aging 2010; 32(2): 200-23.

[58] Holroyd E. Developing a cultural model of caregiving obligations for elderly Chinese wives. West J Nurs Res 2005; 27: 437-56.

[59] Van de Mortel TF. Faking it: Social desirability response bias in self-report research. Aust J Adv Nurs 2008; 25(4): 40-8. 